\title{
The Effects of Liver Function Damage after Thermal Ablation on the Prognosis of HCC Patients and Its Prediction
}

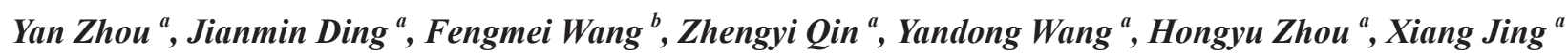 \\ ${ }^{a}$ Department of Ultrasound, Tianjin Institute of Hepatobiliary Disease, Tianjin Key Laboratory of Extracorporeal Life Support for \\ Critical Diseases, Artificial Cell Engineering Technology Research Center; ${ }^{b}$ Department of Gastroenterology and Hepatology, Tianjin \\ Third Central Hospital, Tianjin, China
}

Received June 03, 2021; revision received June 23, 2021; accepted June 25, 2021

Objective: To investigate the influence of liver function damage in patients with hepatocellular carcinoma (HCC) after microwave ablation (MWA) on the prognosis and to establish an algorithm to predict liver function damage.

Methods: This is a retrospective study. A total of 745 patients were enrolled and classified into the modeling group and the validation group. The risk factors of liver function damage were analyzed by using logistic regression model. The $\beta$ coefficients derived from a logistic analysis were used to calculate the risk score. Diagnostic accuracy based on risk score was assessed by the area under the curve (AUC).

Results: 69 patients in the modeling group suffered from liver function damage. The 1-,3-,5- and 7-year progression free survival were $69.4 \%, 37.2 \%, 22.6 \%$ and $12.4 \%$ for patients without liver function damage and $69.5 \%, 33.0 \%, 14.4 \%$ and $0 \%$ for patients with liver function damage, respectively $(P=0.537)$. The 1-, 3-, 5- and 7-year overall survival were $94.3 \%, 75.9 \%, 61.5 \%$ and $54.5 \%$ for patients without liver function damage and $81.3 \%, 57.1 \%, 42.7 \%$ and $42.7 \%$ for those with liver function damage $(P=0.004)$, respectively. The AUC of the risk score for predicting liver function damage was 0.798 for the modeling group and 0.832 for the validation group. The sensitivity and specificity of the risk score to identify liver function damage was $65.2 \%$, $84.4 \%$ in the modeling group and $60.6 \%, 88.3 \%$ in the validation group, respectively.

Conclusion: Liver function damage after MWA is unfavorable the long-term prognosis. The prediction algorithm based on five risk factors is robust and can be used to predict possible liver function damage.

Key words: Microwave ablation; Hepatocellular carcinoma; Liver function damage; Prediction algorithm

Advanced Ultrasound in Diagnosis and Therapy 2021; 02: 080-086

DOI: 10.37015/AUDT.2021.210017

$\mathrm{H}$ epatocellular carcinoma (HCC) is one of the most common malignancy around the world with a high morbidity and mortality $[1,2]$. In recent years, local thermal ablation was used as the radical treatment method in the management of $\mathrm{HCC}$ and can improve the prognosis of patients [3-5]. In clinical practice, the complication is major concern in such mini- invasive therapy [6]. Liver function damage, however, is a common complication after thermal ablation. This damage, however, is still not well studied because serious liver dysfunction is rare and it hardly ever results in death [7]. Previous studies [8-10] demonstrate that liver function damage after thermal ablation is related to liver reserve function, blood platelet or ablation volume

\footnotetext{
* Corresponding author: Department of Ultrasound, Tianjin Institute of Hepatobiliary Disease, Tianjin Key Laboratory of Extracorporeal Life Support for Critical Diseases, Artificial Cell Engineering Technology Research Center, No.83 Jingtang Road, Hedong District, Tianjin, 300170, China

e-mail:djmzyn1982@sina.com unrestricted use, distribution and reproduction in any medium provided that the original work is properly attributed.
} 
and that patients can recover within one month in most cases. The influence of liver function damage on long term outcome, however, is still unclear.

With the extensive use of thermal ablation, several assisting methods can be adopted to prevent visceral organ from thermal damage or other major complication $[11,12]$. Different from other complications, liver function damage is relevant to several factors, such as liver reserve function, tumor location, size and number. It can hardly be avoided by using assisting methods during the procedure. The best way to decrease the incidence of liver dysfunction is strictly performing thermal ablation according to the indications. Therefore, we establish an algorithm based on risk factors after MWA to predict the risk of liver function damage and further help to select suitable candidate for thermal ablation in patients with HCC.

\section{Patients and Methods}

\section{Patients}

This retrospective study was approved by Tianjin Third Central Hospital Institutional Review Board.
From August 2013 to October 2016, patients with HCC undergoing MWA meeting the following inclusion criteria were enrolled in this study: (1) they were diagnosed with HCC and underwent MWA; (2) the diameters of nodules were not larger than $5 \mathrm{~cm}$; (3) the number of nodules were not more than 5; (4) they did not suffer from portal thrombus or extrahepatic metastases; (5) they had liver cirrhosis and a liver function of ChildPugh classification A or B; (6) the level of platelet counts was larger than $50 \times 10^{9} / \mathrm{L}$ or the international normalized ratio was less than or equal to 1.7 ; (7) they did not receive any other invasive treatment, such as PEI, TACE, RFA, during preoperative and postoperative period; (8) they did not suffer from non-liver related serious complications after MWA. A total of 745 patients were included in our study (Fig.1). The diagnoses were reconfirmed by ultrasound guided percutaneous biopsy. All procedures performed in this study involving human participants were in accordance with the ethical standards of the institutional and/or national research committee and with the 1964 Helsinki declaration and its later amendments or comparable ethical standards. The informed consents of the patients were waived.

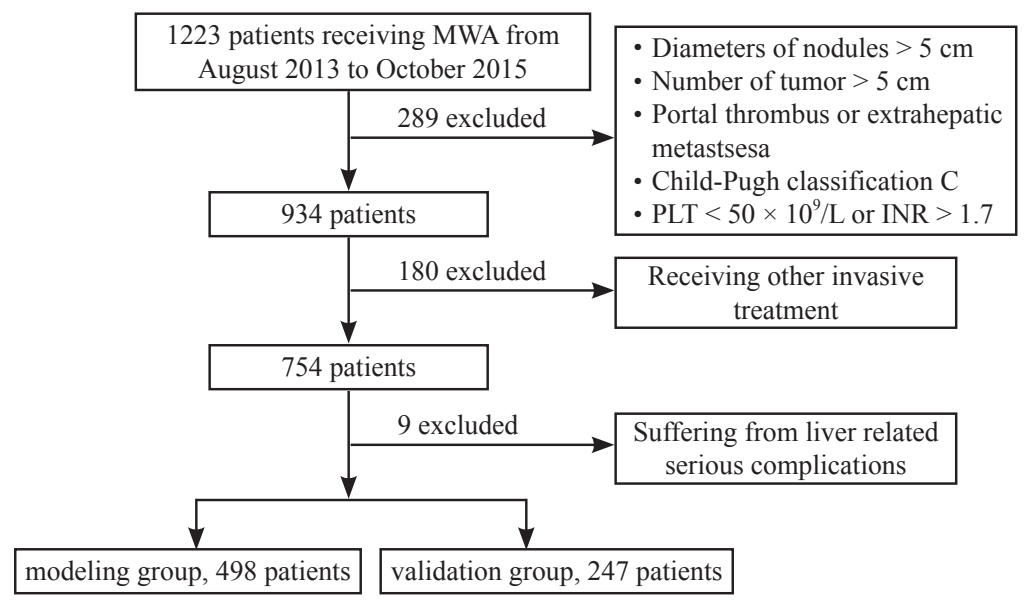

Figure 1 Flow chart describing the inclusion criteria of this study. PLT, platelet; INR, international normalized ratio.

\section{MWA ablation}

Philips IU-22 (Philips, Bothell, WA, USA) and Aloka 5000 (Aloka, Tokyo, Japan) ultrasound systems with 1.0-5.0 $\mathrm{MHz}$ convex array probe were used to guide and monitor the thermal ablation procedures. MWA procedures were carried out using MTC-3 microwave therapy instrument (Forsea Microwave \& Electronic Research Institute, Nanjing, China) with a frequency of 2,450 MHz and an output power of 50-100 W. The antenna was a $14 \mathrm{G}$ unipolar cooled-shaft needle with a $15 \mathrm{~cm}$ length and a $1.5 \mathrm{~cm}$ active tip. The ablation time depended on the size, shape and location of the tumor as well as the coagulation effects. The safe margin for complete ablation was at least $0.5 \mathrm{~cm}$ beyond the tumor boundary (except the tumor close to visceral organ or important tissues). All the procedures were performed by the same radiologist with more than 15 years' experience of thermal ablation.

\section{Follow-up}

The contrast-enhanced CT or MRI was used to evaluate the efficacy one month after treatment. Complete ablation was defined as the postoperative hypoattenuation/ hypointensities in all the phases of the CECT or CEMRI. 
Ultrasound or contrast enhanced ultrasound scans were performed every two to three months. Blood tests for liver function, complete blood count and prothrombin time (PT) were routinely performed along with imaging. Contrast enhanced CT scans were repeated with an interval of six to twelve month. Tumor recurrence was defined as the occurrence of the hyper vascular in arterial phase and wash out in portal or delay phase.

\section{Establishment and validation of the risk score for prediction of liver function damage}

Patients were classified into the modeling group (498 patients from August 2013 to August 2015) and the validation group (247 patients from September 2015 to October 2016). The clinical characteristics of the two groups are shown in table 1 . Seven candidate risk factors, including the relative position between the tumor and $\mathrm{PV}$, number of tumors, ablation volume, aspartate aminotransferase-to-platelet ratio index (APRI), MELD score, platelet count (PLT) and Child-Pugh classification before treatment, were taken into account in the prediction algorithm. All of the candidate risk factors were categorized (Table 2) [13]. The liver function damage was defined as an increase of Child-Pugh score by two three days after MWA. It should be noted that liver function damage defined in this study differs from the post-ablation syndrome, which usually includes lowgrade fever and flulike symptoms within the first 24-48 hours after thermal ablation.

Table 1 Baseline characteristics of the modeling and validation group before MWA ablation

\begin{tabular}{|c|c|c|c|}
\hline Item & Modeling group & Validation group & $P$ value \\
\hline Number of patients & 598 & 247 & \\
\hline Number of tumors & 721 & 359 & \\
\hline Gender $(\mathrm{M} / \mathrm{F})$ & $374 / 124$ & $190 / 57$ & 0.585 \\
\hline Age (years) & $59.56 \pm 9.07$ & $60.02 \pm 8.84$ & 0.497 \\
\hline Etiology & & & 0.477 \\
\hline HBV & 410 & 209 & \\
\hline $\mathrm{HCV}$ & 55 & 28 & \\
\hline Alcoholic & 9 & 4 & \\
\hline Other & 24 & 6 & \\
\hline Tumor size $(\mathrm{cm})$ & $2.39 \pm 0.82$ & $2.20 \pm 0.83$ & 0.007 \\
\hline Child-pugh classification (A/B) & $432 / 66$ & $220 / 27$ & 0.367 \\
\hline $\operatorname{ALB}(g / L)$ & $39.84 \pm 5.99$ & $40.88 \pm 5.66$ & 0.023 \\
\hline $\operatorname{ALT}(\mathrm{U} / \mathrm{L})$ & $32.68 \pm 22.01$ & $31.32 \pm 29.26$ & 0.476 \\
\hline $\operatorname{AST}(\mathrm{U} / \mathrm{L})$ & $36.14 \pm 22.54$ & $33.03 \pm 22.17$ & 0.075 \\
\hline TBIL $(\mu \mathrm{mol} / \mathrm{L})$ & $18.87 \pm 10.40$ & $20.91 \pm 13.37$ & 0.022 \\
\hline $\operatorname{PLT}\left(10^{9} / \mathrm{L}\right)$ & $108.16 \pm 58.66$ & $115.59 \pm 68.80$ & 0.126 \\
\hline
\end{tabular}

HBV, Hepatitis B virus; HCV, Hepatitis c virus; ALB, Albumin; ALT, Alanine aminotransferase; AST, Aspartate aminotransferase; TBIL, Total bilirubin; PLT, Platelet.

Table 2 The candidate risk factors of liver function damage

\begin{tabular}{|c|c|}
\hline Factor & Categorized \\
\hline Relative position between the tumor and $\mathrm{PV}^{\mathrm{a}}$ & $\begin{array}{l}\text { (1) far away from PV; (2) close to the third branch of PV }(<5 \mathrm{~mm}) \text {; (3) close to the second branch of PV } \\
(<5 \mathrm{~mm}) \text {; (4) close to the first branch of PV }(<5 \mathrm{~mm}) \text {. }\end{array}$ \\
\hline Number of tumor & (1) solitary tumor; (2) 2 or 3 tumors; (3) more than 3 tumors \\
\hline Ablation volume $^{\mathrm{b}}$ & $(1)<22.5 \mathrm{~cm}^{3} ;(2) \geqslant 22.5 \mathrm{~cm}^{3}$ \\
\hline APRI b & (1) $\mathrm{APRI} \leqslant 0.8$; (2) $0.8<\mathrm{APRI} \leqslant 1.6$; (3) APRI $>1.6$ \\
\hline MELD score ${ }^{\mathrm{b}}$ & (1) MELD score $<25$; (2) $25 \leqslant$ MELD score $<30$; (3) MELD score $>30$ \\
\hline PLT b & (1) $\mathrm{PLT}<110 \times 10^{9} / \mathrm{L} ;(2) \mathrm{PLT} \geqslant 110 \times 10^{9} / \mathrm{L}$ \\
\hline Child Classification & (1) Child A; (2) Child B \\
\hline
\end{tabular}

APRI, Aspartate aminotransferase-to-platelet ratio index; MELD, Model for end-stage liver disease; PLT, Platelet.

${ }^{\mathrm{a}}$ : tumors close to the branch of PV was defined as the distance of tumor and the branch of PV is shorter than $5 \mathrm{~mm}$; ${ }^{\mathrm{b}}$ : the cut-off values are determined by ROC curve. 
The above seven factors were fitted into a logistic regression model. The risk score was created by multiplying all the $\beta$ coefficients of the significant variables by 10 , rounding them to the nearest integer and sum them up. A receiver operating characteristic (ROC) curve was conducted for the modeling group and the validation group. The cut-off point was found, at which the sum of the sensitivity and the specificity is maximal.

\section{Statistical Analysis}

Continuous variables were shown as mean \pm standard deviation. Categorical variables were presented by frequencies and percentages. Comparisons of the parameters were performed using the Student's t-test or chi-square test. The logistic regression model was used to screen the risk factors. The $\beta$ coefficients of the significant variables were used to calculate risk score. Diagnostic accuracy of the risk assessment algorithm was assessed by the area under the curve (AUC). The Kaplan Meier analysis was used to calculate the progression free survival (PFS) and overall survival (OS) of the modeling group. The log-rank tests were used to compare PFS or OS in different groups.

\section{Results}

Complete ablation was achieved in 1039 of 1080 tumors. The rates of complete ablation for the modeling and validation groups were $95.6 \%(689 / 721)$ and $97.5 \%$ (340/359), respectively $(P=0.118)$. No procedure related

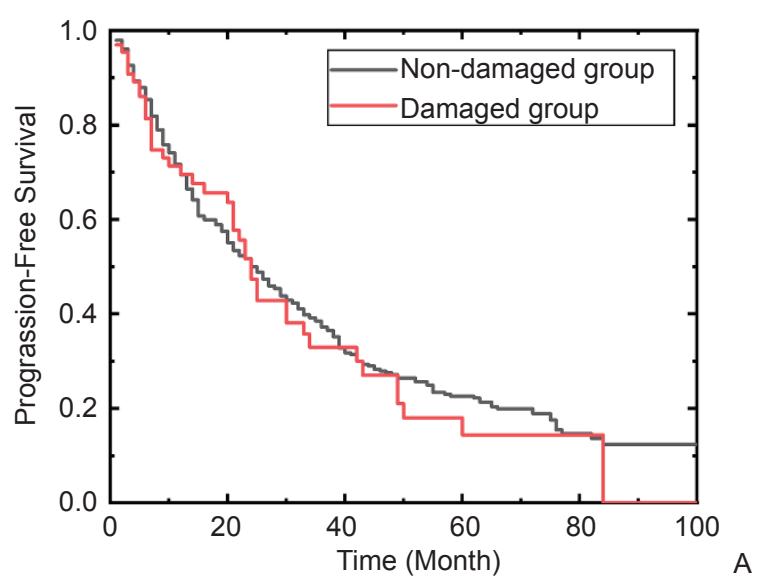

death in both groups was found, but one patient died due to the multiple organ failure one month after treatment. The incidences of liver function damage in the modeling and validation groups were $13.9 \%(69 / 498)$ and $13.4 \%$ (33/247), respectively $(P=0.853)$.

\section{Influence of Liver Function Damage on Prognosis}

The median follow-up is 38 months (ranging from 1 to 93 months) in the modeling group. The patients were further classified into the liver function damage subgroup and non-damage subgroup. A total of $33.3 \%$ patients in the non-damage subgroup and $39.1 \%$ patients in the damage subgroup died during the follow-up. $69.7 \%$ and $65.1 \%$ had tumor progression in non-damage and damage subgroups. The 1-, 3-, 5- and 7-year PFS were $69.4 \%, 37.2 \%, 22.6 \%$ and $12.4 \%$ for the non-damage subgroup and $69.5 \%, 33.0 \%, 14.4 \%$ and $0 \%$ for the damage subgroup respectively $(P=0.573)$. The $1-, 3-$, 5 - and 7-year OS are $94.3 \%, 75.9 \%, 61.5 \%$ and $54.5 \%$, respectively, for the non-damage subgroup, higher than the those for the damage subgroup $(81.3 \%, 57.1 \%$, $42.7 \%$ and $42.7 \%, P=0.004$ ) (Fig. 2).

\section{Prediction Algorithm for Liver Function Damage}

The results of univariate logistic regression analysis showed that all of the seven candidate factors had effects on liver function damage, but the Child-Pugh classification, PLT and MELD score failed to be statistically significant in the multiple logistic regression analysis (Table 3).

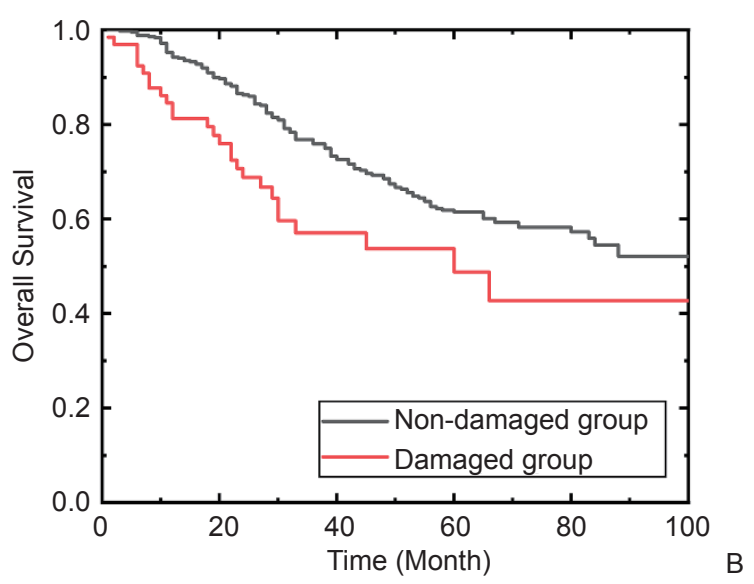

Figure 2 (A) the progression free survival (PFS); (B) the overall survival (OS) in the liver function damage and non-damage subgroup.

The $\beta$ coefficients of all the variables and the corresponding risk score are shown in table 4 . The risk score for liver function damage of patients is the sum of the scores of all the significant variables (the ChildPugh classification is incorporated into the prediction algorithm considering the clinical significance).

The ROC curve for the risk score for the modeling group are depicted in figure 3 . The AUC is 0.798 . At a cut-off value of 24 , the sensitivity and specificity of the risk score in the modeling group is $65.2 \%$ and 
$84.4 \%$, respectively. When applying the algorithm to the validation group, it gives an AUC of 0.832. At the same cut-off value, the sensitivity is $60.6 \%$ and the specificity is $88.3 \%$ (Fig. 3).

Table.3 Logistic regression analysis for liver function damage after MWA

\begin{tabular}{|c|c|c|c|c|c|c|}
\hline \multirow{2}{*}{ Variable } & \multicolumn{3}{|c|}{ Univariate analysis } & \multicolumn{3}{|c|}{ Multiple analysis } \\
\hline & $\beta$ coefficients & Odds ratio & $P$ value & $\beta$ coefficients & Odds ratio & $P$ value \\
\hline \multicolumn{7}{|c|}{ Relative position between the tumor and PV } \\
\hline Far away & 1.00 & 1.00 & & 1.00 & 1.00 & \\
\hline Close to the third branch & 1.36 & 3.89 & $<0.001$ & 1.40 & 4.09 & $<0.001$ \\
\hline Close to the second branch & 2.40 & 11.01 & $<0.001$ & 2.83 & 16.93 & $<0.001$ \\
\hline Close to the first branch & 1.55 & 4.72 & 0.079 & 1.24 & 3.48 & 0.198 \\
\hline \multicolumn{7}{|l|}{ Number of tumors } \\
\hline 1 & 1.00 & 1.00 & & 1.00 & 1.00 & \\
\hline 2 or 3 & 0.92 & 2.50 & 0.010 & 0.77 & 2.17 & 0.019 \\
\hline More than 3 & 2.10 & 8.14 & $<0.001$ & 1.56 & 4.76 & 0.027 \\
\hline Ablation volume & 1.10 & 3.02 & $<0.001$ & 0.74 & 2.09 & 0.025 \\
\hline \multicolumn{7}{|l|}{ APRI } \\
\hline$\leqslant 0.8$ & 1.00 & 1.00 & & 1.00 & 1.00 & \\
\hline$\leqslant 1.6$ & 0.68 & 1.98 & 0.059 & 0.28 & 1.33 & 0.544 \\
\hline$>1.6$ & 1.87 & 6.48 & $<0.001$ & 1.62 & 5.05 & 0.001 \\
\hline Platelet & 1.11 & 3.03 & $<0.001$ & 0.13 & 1.14 & 0.780 \\
\hline \multicolumn{7}{|l|}{ MELD score } \\
\hline$<25$ & 1.00 & 1.00 & & 1.00 & 1.00 & \\
\hline$<30$ & 1.04 & 2.84 & 0.001 & 0.91 & 2.45 & 0.049 \\
\hline$\geqslant 30$ & 1.56 & 4.77 & $<0.001$ & 1.08 & 2.93 & 0.050 \\
\hline
\end{tabular}

APRI, Aspartate aminotransferase-to-platelet ratio index; MELD, Model for end-stage liver disease.

Table $4 \beta$ coefficients of each variable and its corresponding risk score

\begin{tabular}{|c|c|c|}
\hline Variable & $\beta$ coefficients & Score \\
\hline \multicolumn{3}{|c|}{ Relative position between the tumor and PV } \\
\hline Far away & & 1 \\
\hline Close to the third branch & 1.40 & 14 \\
\hline Close to the second branch & 2.83 & 28 \\
\hline Close to the first branch & 1.24 & 12 \\
\hline Number of tumors & & 1 \\
\hline \multicolumn{3}{|l|}{1} \\
\hline 2 or 3 & 0.77 & 8 \\
\hline More than 3 & 1.56 & 16 \\
\hline \multicolumn{3}{|l|}{ Ablation volume } \\
\hline$<22.5 \mathrm{~cm}^{3}$ & & 1 \\
\hline$\geqslant 22.5 \mathrm{~cm}^{3}$ & 0.74 & 7 \\
\hline \multicolumn{3}{|l|}{ Child-Pugh Classification } \\
\hline Child A & & 1 \\
\hline Child B & 0.34 & 3 \\
\hline \multicolumn{3}{|l|}{ APRI } \\
\hline$\leqslant 0.8$ & & 1 \\
\hline$\leqslant 1.6$ & 0.28 & 3 \\
\hline$>1.6$ & 1.62 & 16 \\
\hline
\end{tabular}

Score, 10 times of $\beta$ coefficients and rounded to the nearest integer; APRI, Aspartate aminotransferase-to-platelet ratio index.

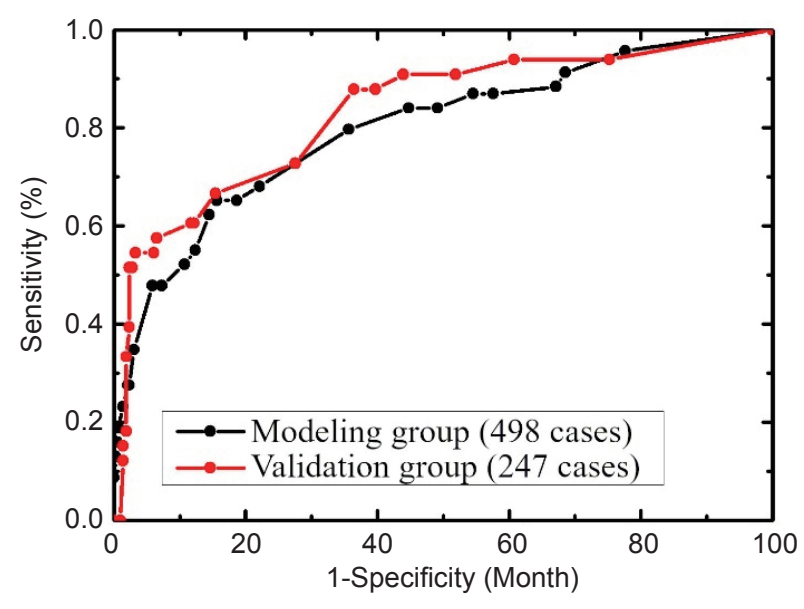

Figure 3 Performance of our algorithm for predicting liver function damage after MWA (black line: the modeling group; red line: the validation group).

\section{Discussion}

Thermal ablation of HCC can induce the damage of normal liver parenchyma due to the safe margin area, "heat sink effect" or occluded blood vessels of liver. Although, serious damage of normal liver parenchyma 
rarely occurred [14], liver function damage, mirrored by the evaluation of liver enzymes and a shift in ChildPugh classification, is a common complication induced by thermal ablation $[8-10,15]$. The results of Li et al. [16] demonstrated that the Child-Pugh score increased from $5.2 \pm 1.3$ to $7.1 \pm 1.5$ three days after trans catheter arterial chemoembolization combined with percutaneous radiofrequency ablation in patients with hepatocellular carcinoma $(P<0.021)$. Lee et al. [9] reported that the Child-Pugh score was significantly increased 12 months after RFA in patients with HCC. Koike et al. [17] also found that the Child-Pugh classification of $7 \%$ patients shifted from A to B, and of $14 \%$ patients shifted from $\mathrm{B}$ to $\mathrm{C}$ one year after percutaneous tumor ablation. In clinical practice, liver function damage after thermal ablation is not serious even without any extra care, because the increased liver enzymes may recover to normal value within 3-7 days and the shifted Child-Pugh classification may return to the level before treatment in several months [8-10]. Patients with liver function of Child-Pugh classification A or B rarely suffer from liver dysfunction after thermal ablation [17]. Therefore, it is generally assumed that liver function damage caused by thermal ablation had a negligible effect on the prognosis [18]. A previous study [17], however, mentioned that the chronic hepatic disease is a risk factor having an effect on prognosis. Thus, we infer that the change of liver function, as an indicator of the progress of hepatic disease, may also influence the long-term outcome of patients with HCC.

In order to prove our hypothesis, we analyzed the effects of liver function damage induced by MWA in 498 patients on the long-term outcome retrospectively. According to the definition of liver function damage after MWA, namely an increase of the score of the ChildPugh score by 2 three days after treatment, a total of 69 patients suffered from this complication. The results of our study show that the 1-, 3-, 5- and7- year PFS are comparable between the liver function damage group and non-damage group, which demonstrates that the liver function damage induced by MWA have no effect on the progression of HCC. In contrast to the results of PFS, patients in the non-damage group gained higher OS. We infer that this result is because: (1) patients with poor liver function were prone to suffer from liver function damage after MWA. The combination of poor liver function and function damage prevents patients from favorable prognosis; (2) Different from the recovery of liver enzymes in a short time, the damage of liver function has a potential effect on the long-term prognosis.

Improving the treatment safety and decreasing the risk of liver function damage is very important in promoting the prognosis for patients. However, liver function damage can hardly be avoided during the ablation. Therefore, determination of whether a patient is suitable for this treatment by strictly obeying its indication is very important. Child-Pugh classification $\mathrm{A}$ or $\mathrm{B}$ is the indication for thermal ablation. Rare liver function damage occurs after therapy when patients are with a relatively good liver reserve function. Previous studies $[9,10,16]$, however, demonstrated that thermal damage of liver function was affected by many factors, apart from Child-Pugh classification of liver function, tumor number, ablation volume and tumor location. Our previous study [13] founds that five risk factors were related to liver function damage after MWA. Therefore, we establish an algorithm based on these factors to predict the risk of liver function damage.

We used logistic regression to select risk factors for the assessment algorithm. The number of tumors, ablation volumes, relative position between the tumor and PV and APRI were selected as the risk factors used to establish the algorithm according to the results of the multivariate logistic regression. It should be pointed that the risk factor should be achieved before the treatment. However, ablation volume can be achieved only after the ablation. Therefore, in practice, ablation volume can be estimated by calculating the volume of a sphere with a radius equal to tumor radius plus a safe margin of $5 \mathrm{~mm}$. The Child-Pugh classification was excluded by the logistic regression with a $\mathrm{p}$ value of 0.432. However, Kuroda et al. [10] and Li et al. [16] reported that patients with Child-Pugh scores larger than 8 are at the high risk of the aggravation of liver dysfunction and may suffer from complications when treated by RFA. Considering the clinical significance of liver function, the Child-Pugh classification before treatment was enrolled additionally. In order to evaluate the performance of the risk assessment algorithm, it was used to predict liver function after thermal ablation both in the modeling group and the validation group. The results show that the AUC are 0.798 in modeling group and 0.832 in validation group. The sensitivity and specificity of the risk score is $65.2 \%$ and $84.4 \%$ in the modeling group, and $60.6 \%$ and $88.3 \%$ in the validation group, respectively, which indicates that our algorithm can predict the risk of liver dysfunction after thermal ablation. Based on the discussion above, we suggest that patients with risk score larger than 24 should not receive MWA before improving the liver function.

There are some limitations in this study. First, prognosis of HCC patients during the long follow-up may be affected by the chronic liver cirrhosis. Second, this study is a single central study. The results of our study need validation by multicenter data. 


\section{Conclusion}

Liver function damage induced by MWA has no effect on the progression of HCC but it is an unfavorable factor for overall survival. Patients with liver function damage after MWA may have poor prognosis. The prediction algorithm based on the Child-Pugh classification before treatment, tumor numbers, ablation volumes, relative position between the tumor and portal vein (PV) and APRI is a robust model for predicting the possibility of liver function damage in patients with HCC after MWA. Therefore, thermal ablation should be carefully performed for the patients with the risk factors of liver function damage. Strategies to improve liver function before and after the ablation should be performed to avoid liver function damage and to improve the prognosis.

\section{Acknowledgements}

This work was supported by Tianjin health and Health Committee (Grant No. ZD20014, MS20017, KJ20170, NQ20033).

\section{Conflict of Interest}

The authors declare no conflict of interest.

\section{References}

[1] Ferlay J, Soerjomataram I, Dikshit R, Eser S, Mathers C, Rebelo M, et al. Cancer incidence and mortality worldwide: sources, methods and major patterns in GLOBOCAN 2012. Int J Cancer 2015;136: E359-E386.

[2] Torre LA, Bray F, Siegel RL, Ferlay J, Lortet-Tieulent J, Jemal A. Global cancer statistics, 2012. CA: Cancer J Clin 2012;65: 87-108.

[3] Yu J, Yu X, Han Z, Cheng Z, Liu F, Zhai H, et al. Percutaneous cooled-probe microwave versus radiofrequency ablation in earlystage hepatocellular carcinoma: a phase III randomised controlled trial. Gut 2017; 66: 1172-1173.

[4] Weinstein JL, Ahmed M. Percutaneous ablation for hepatocellular carcinoma. Am J Roentgenol 2018; 201:1368-1375.

[5] Poggi G, Ricardi A, Quaretti P, Teragni C, Angelo D, Amatu A, et. al. Complications of percutaneous radiofrequency thermal ablation of primary and secondary lesions of the liver. Anticancer Res 2007; 27: 2911- 2916.

[6] Bertot LC, Sato M, Tateshi R, Yoshida H, Koike K. Mortality and complication rates of percutaneous ablative techniques for the treatment of liver tumors: a systematic review. Eur Radiol 2011; 21:
2584- 2596.

[7] Liang P, Wang Y, Yu XL, Dong B. Malignant liver tumors: treatment with percutaneous microwave ablation-complications among cohort of 1136 patients. Radiology 2009; 25: 933-940.

[8] Jiang K, Dong J, Zhang W, Liu Y, Su M, Zhao X, et al. Effect of oneoff complete tumor radiofrequency ablation on liver function and postoperative complication in small hepatocellular carcinoma. Eur J Surg Oncol 2014; 40: 576-583.

[9] Lee HS, Park SY, Kim SK, Kweon YO, Tak WY, Cho CM, et al. Thrombocytopenia represents a risk for deterioration of liver function after radiofrequency ablation in patients with hepatocellular carcinoma. Clin Mol Hepatol 2012; 18:302-308.

[10] Kuroda H, Kasai K, Kakisaka K, Yasumi Y, Kataoka K, Ushio A, et al. Changes in liver function parameters after percutaneous radiofrequency ablation therapy in patients with hepatocellular carcinoma. Hepatol Res 2010; 40: 550-554.

[11] Hsieh YC, Limquiaco JL, Lin CC, Chen WT, Lin SM. Radiofrequency ablation following artificial ascites and pleural effusion creation may improve outcomes for hepatocellular carcinoma in high-risk locations. Abdom Radiol 2019; 44:1141-1151.

[12] Huang H, Liang P, Yu X, Cheng Z, Han Z, Yu J, et al. Safety assessment and therapeutic efficacy of percutaneous microwave ablation therapy combined with percutaneous ethanol injection for hepatocellular carcinoma adjacent to the gallbladder. Int $J$ Hyperthermia 2015; 31: 40-47.

[13] Zhou Y, Jing X, Ding J, Jing X, Wang F, Wang Y, et al. Risk factors of liver dysfunction induced by microwave ablation in patients with hepatocellular carcinoma. Ultrasound in Medicine \& Biology 2017, 43: S162-S162.

[14] Wake T, Tatesjo R, Nakagomi R, Fujiwara N, Kinoshita MN, Nakatsuka T, et. al. Ischemic complications after percutaneous radiofrequency ablation of liver tumors Liver volume loss and recovery. Hepatol Res 2019; 49: 453-461.

[15] Liu R, Li K, Luo H, Zhang W, Zhang T, Gao M, et al. Ultrasoundguided percutaneous microwave ablation for small liver cancers adjacent to large vessels: Long-term outcomes and strategies. Oncol Transl Med 2017; 3: P57-P64.

[16] Li JX, Wu H, Huang JW, Zeng Y. The influence on liver function after transcatheter arterial chemoembolization combined with percutaneous radiofrequency ablation in patients with hepatocellular carcinoma. J Formos Med Assoc 2012; 111:510-515.

[17] Koike Y, Yoshida H, Shiina S, Takuma T, Obi S, Sato S, et al. Changes in hepatic functional reserve after percutaneous tumor ablation for hepatocellular carcinoma: long-term follow up for 227 consecutive patients with a single lesion. Hepatol Int 2007; 1:295-301.

[18] Huang Q, Xu E, Tan L, Zeng Q, Zheng R, Li K. Thermal ablation of hepatocellular carcinoma in patients with abnormal coagulation function. Int J Hyperthermia 2018; 34: 1038-1043. 